\title{
Consideration of the Phase Plane of the "Directional" Grouping Wave
}

\author{
by Ben T. Nohara*, Member
}

\begin{abstract}
Summary
Goda and Longuet-Higgins originally reviewed the grouping wave. However, we can find few studies on the grouping wave in the literature after them. It has been known that the grouping wave is strongly related to the break of mooring lines of a vessel in a port. In the mega-float case, especially, this problem will become a severe one due to no past experience in real design.

This paper discusses the characteristics of the grouping wave. The "directional" grouping wave is defined from the multi-directional regular wave and also the "complex" grouping wave is reduced from the multi-directional irregular wave.
\end{abstract}

\section{Introduction}

Goda $^{1)}$ and Longuet-Higgins ${ }^{2)}$ originally reviewed the grouping wave (which has been referred by the "wave group" in their paper). However, we can find few studies on the grouping wave in the literature ${ }^{3 / 4) 5)}$ after them. It has been known that the grouping wave is strongly related to the break of mooring lines of a vessel in a port ${ }^{6)}$. In the mega-float ${ }^{7)}$ case, especially, this problem will become a severe one due to no past experience in real design.

A "swell"* is a mostly simple case of the grouping wave. A "swell" is synthesized by two component waves in which frequencies are very close. The motion of a "swell" has a rapid oscillation with slowly varying amplitude. The frequency of individual waves of a "swell" is high: the half of the sum of two frequencies, but the frequency of its envelope is low: the half of the difference of two frequencies.

Here, let us define the grouping wave precisely. The grouping wave is defined by the irregular wave specified by the spectrum of the wave envelope. The normal irregular wave does not have the specific wave envelope. Using the expression of the signal processing technique, the grouping wave is the wave of which

Note: This paper suggests the term: a "swell" for the superposition of two close, frequency component waves. The term: a "directional" swell is also used for the superposition of two close, directional component waves in the same manner.

* Mitsubishi Heavy Industries, Ltd.

Received 11th Jan. 1999

Read at the Spring meeting 12, 13th May 1999 phase modulated carrier wave is modulated by the amplitude $^{8)}$. Time series of the amplitude modulation profiles the wave envelope. So, the grouping wave can be generated by the product of the carrier wave with the original spectrum of the irregular wave and the amplitude modulation with the wave envelope spectrum $^{9)}$.

In the reference ( 9 ), the normal irregular wave and the grouping wave are generated by this method in a test basin and the mooring characteristics of a pontoon model are observed. The fact that the remarkable and low-frequency sway motion of a model is produced by the grouping wave, especially, of which envelope period is a half of sway resonance of a model, is cleared by the experiments. Namely, the sub-harmonic motion subject to the envelope period has occurred in the sway motion.

Moreover, it is known that the grouping wave has the chaos-like feature $^{10)}$. To analyze this feature, the continuing research is required.

This paper discusses the characteristics of the grouping wave. The "directional" grouping wave is defined from the multi-directional regular wave and also the "complex" grouping wave is reduced from the multidirectional irregular wave.

The following section describes the survey of the grouping wave including theoretical background. Section 3 expands the normal grouping wave to the "directional" one. Section 4 deals with the "complex" grouping wave which is created from the very natural wave, so-called the "short crested" wave. In the final section, the concluding remark is presented.

\section{Survey of the Grouping Wave}

\subsection{Theoretical background ${ }^{11}$}

We shall start by looking at the concept of the wave 
envelope. The surface profile $\eta_{\sigma}(x, y, t)$ of the irregular ocean wave is formulated in rectangular coordinates system $(x, y)$ using low amplitude, linear theory as follows :

$$
\begin{aligned}
\eta_{\sigma}(x, y, t)= & \sum_{n=1}^{N} a_{n} \cos \left(\sigma_{n} t+k_{n} x \cos \theta\right. \\
& \left.+k_{n} y \sin \theta+\varepsilon_{n}\right)
\end{aligned}
$$

where the subscription $n$ indicates the values of $n$-th frequency.

Equation 1 shows the uni-directional irregular wave which consists of $N$ frequency component waves with propagation direction $\theta$. Here, $a, \sigma, k, \varepsilon$ and $t$ denote amplitude, angular frequency, wave number, random phase lag defined from 0 to $2 \pi$ and time, respectively. Let the frequency spectrum of this wave be $S(\sigma)$, then $a_{n}$ is written by the following equation.

$$
a_{n}=\sqrt{2 S\left(\sigma_{n}\right) \Delta \sigma_{n}}
$$

Here, $\Delta \sigma$ represents the defined frequency bandwidth in the effectively spectral domain. That is, the given wave spectrum $S(\sigma)$ can be realized by Eq. 2. Moreover, wave number $k_{n}$ is a solution of the dispersion relation.

$$
\sigma_{n}^{2}=g k_{n} \tanh k_{n} h
$$

Here, $g$ and $h$ indicate the acceleration due to gravity and uniform water depth, respectively.

Let us consider the envelope of Eq. 1. Using the concept of modulation in the signal processing, Eq. 1 can be generally rewritten as follows:

$$
\eta_{\sigma}(x, y, t)=r_{\sigma}(x, y, t) \cos \psi_{\sigma}(x, y, t)
$$

Here, $r_{\sigma}(x, y, t)$ and $\psi_{\sigma}(x, y, t)$ mean amplitude modulation and phase modulation as the function of time $t$ and coordinates variables $x$ and $y$, respectively. Therefore, we can consider that amplitude modulation $r_{\sigma}(x$, $y, t)$ describes the feature of the wave envelope surface and also $\cos \psi_{\sigma}(x, y, t)$ characterizes the carrier surface of the irregular wave $\eta_{\sigma}(x, y, t)$.

The wave envelope is obtained by the following $\operatorname{method}^{8)}$. If the signal :

$$
\xi_{\sigma}(x, y, t)=r_{\sigma}(x, y, t) \sin \psi_{\sigma}(x, y, t)
$$

is obtained, then it follows that

$$
r_{\sigma}(x, y, t)=\sqrt{\eta_{\sigma}^{2}(x, y, t)+\xi_{\sigma}^{2}(x, y, t)} .
$$

In general, the signal $\xi(x, y, t)$ which is rotated by $\pi / 2$ in terms of time phase in Eq. 1 is earned using Hilbert transform ${ }^{12)}$ as follows :

$$
\xi_{\sigma}(x, y, t)=\frac{1}{\pi} \int_{-\infty}^{\infty} \frac{\eta_{\sigma}(x, y, \tau)}{t-\tau} d \tau
$$

Phase modulation $\phi(x, y, t)$ is obtained by

$$
\psi_{\sigma}(x, y, t)=\tan ^{-1} \frac{\xi_{\sigma}(x, y, t)}{\eta_{\sigma}(x, y, t)} .
$$

\subsection{Grouping wave}

The grouping wave is defined by the irregular wave specified by the spectrum of the wave envelope. The spectrum of the wave envelope is called "the secondary spectrum", compared with the individual wave spectrum: "the primary spectrum". We had considered the primary spectrum only for a long time. However, we have recently known the secondary spectrum is also important in engineering point of view. For example, the secondary spectrum acts on the significant role of the mooring systems of a vessel in a port. That is, the long period wave which causes the break of mooring lines in spite of relatively calm ocean conditions can be induced by the secondary spectrum.

\subsection{Swell}

A swell is a mostly simple and special case of a grouping wave. Barber and Ursell ${ }^{13)}$ showed swell waves of about 20 seconds period which had traveled more than 1,500 miles across the North Atlantic taking two days, and on a different occasion, swell waves of about 14 seconds period which had traveled 6,000 miles from the Southern Ocean taking twelve days.

A swell is formulated by the summation of two component frequency waves. In Eq. 1 , let $N=2, a_{1}=a_{2}$ $=a$ and $\varepsilon_{1}=\varepsilon_{2}=0$, then it follows that

$$
\begin{aligned}
\eta_{\sigma}(x, y, t)= & a \cos \left(\sigma_{1} t+k_{1} x \cos \theta+k_{1} y \sin \theta\right) \\
+ & a \cos \left(\sigma_{2} t+k_{2} x \cos \theta+k_{2} y \sin \theta\right) \\
= & 2 a \cos \left(\frac{\sigma_{1}-\sigma_{2}}{2} t+\frac{k_{1}-k_{2}}{2} x \cos \theta\right. \\
+ & \left.\frac{k_{1}-k_{2}}{2} y \sin \theta\right) \\
& \times \cos \left(\frac{\sigma_{1}+\sigma_{2}}{2} t+\frac{k_{1}+k_{2}}{2} x \cos \theta\right. \\
+ & \left.\frac{k_{1}+k_{2}}{2} y \sin \theta\right)
\end{aligned}
$$

A point to notice is that if the values of $\sigma_{1}$ and $\sigma_{2}$ are very close, the motion of Eq. 9 has a rapid oscillation with slowly varying amplitude. The first cosine function of the second expression of Eq. 9 corresponds to the wave envelope surface and the second cosine function presents the carrier surface of the wave $\eta(x, y, t)$.

Figure 1 shows the time histories of a swell observed at a fixed place, in which the angular frequencies are 0. $314[\mathrm{rad} / \mathrm{sec}]$ and $0.57[\mathrm{rad} / \mathrm{sec}]$ and the wave propagation is $\pi / 2$ [rad]. The dotted line represents the wave envelope of a swell. Figure 2 demonstrates the surface

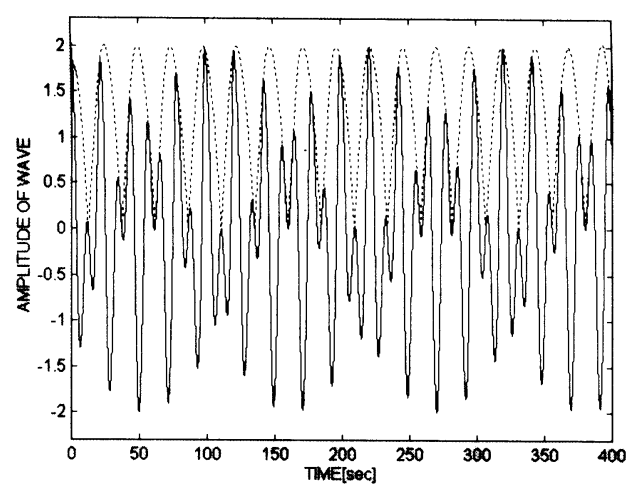

Fig. 1 Time histories of a swell observed at fixed place, in which the angular frequencies are 0.314 $[\mathrm{rad} / \mathrm{sec}]$ and $0.57[\mathrm{rad} / \mathrm{sec}]$ and the wave propagation is $\pi / 2$ [ rad]. The dotted line represents the wave envelope of a swell. 


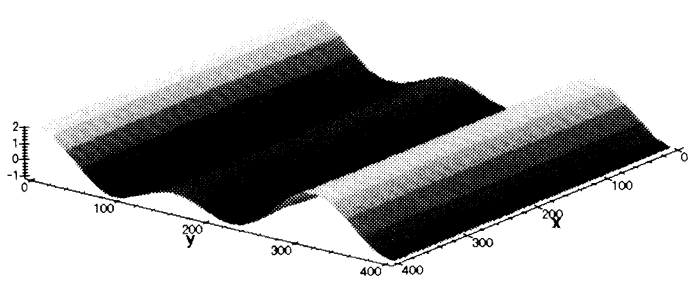

Fig. 2 Surface of a swell of Fig. 1.

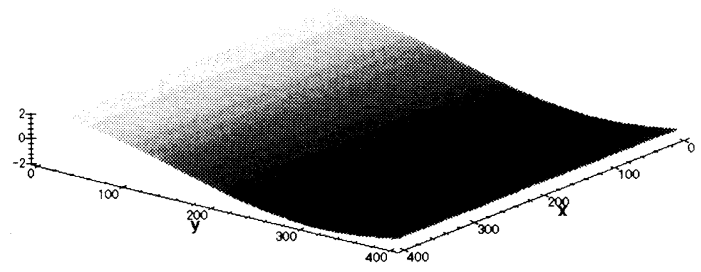

WAVE ENVELOPE SURFACE

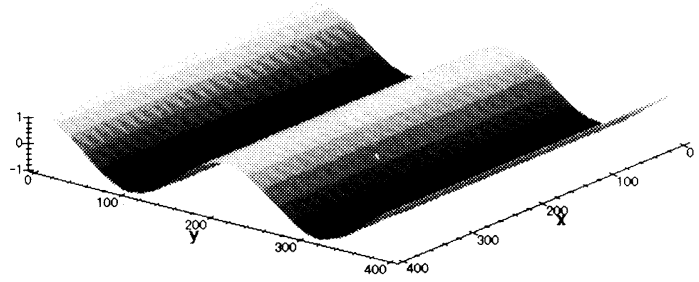

CARRIER SURFACE

Fig. 3 Decomposed surfaces: the wave envelope surface and the carrier surface of Fig. 2 (A "frequency" swell).

of a swell of Fig. 1. Figure 3 shows the decomposed surfaces: the wave envelope surface and the carrier surface.

\section{3. “Directional" Grouping Wave}

\section{1 Multi-directional wave and its envelope}

The surface profile $\eta_{\theta}(x, y, t)$ of the directional wave is formulated by the following equation in a similar way of section 2 .

$$
\begin{aligned}
\eta_{\theta}(x, y, t)= & \sum_{m=1}^{M} a_{m} \cos \left(\sigma t+k x \cos \theta_{m}\right. \\
& \left.+k y \sin \theta_{m}+\varepsilon_{m}\right)
\end{aligned}
$$

Here, the subscription $m$ means the $m$-th direction. This wave: the multi-directional wave has a single, angular frequency $\sigma$. However, Eq. 10 indicates that $M$ directional component waves exist. Let the directional distribution function of this wave be $G(\theta)$, then $a_{m}$ is written by the following equation assuming that the frequency, power spectrum of Eq. 10:S( $\sigma)=1$.

$$
a_{m}=\sqrt{2 G\left(\theta_{m}\right) \Delta \theta_{m}}
$$

Here $\Delta \theta$ represents the defined directional bandwidth in the effectively directional region. Let us consider the envelope of Eq. 10. In a similar way of the case of the uni-directional irregular wave, Eq. 10 can be rewritten as the product of the wave envelope and the carrier as follows :

$$
\begin{aligned}
& \eta_{\theta}(x, y, t)=\sqrt{\eta_{\theta}^{2}(x, y, t)+\xi_{\theta}^{2}(x, y, t)} \cos \psi_{\theta}(t) \\
& \xi_{\theta}(x, y, t)=\frac{1}{\pi} \int_{-\infty}^{\infty} \frac{\eta_{\theta}(x, y, \tau)}{t-\tau} d \tau \\
& \psi_{\theta}(x, y, t)=\tan ^{-1} \frac{\xi_{\theta}(x, y, t)}{\eta_{\theta}(x, y, t)}
\end{aligned}
$$

The wave envelope is obtained by easy calculation as follows.

$$
\begin{aligned}
& \sqrt{\eta_{\theta}^{2}(x, y, t)+\xi_{\theta}^{2}(x, y, t)} \\
& =\sqrt{\left\{\sum_{m=1}^{M} a_{m} \cos \left\{f_{m}(x, y)\right\}\right\}^{2}+\left\{\sum_{m=1}^{M} a_{m} \sin \left\{f_{m}(x, y)\right\}\right\}^{2}} \\
& =r_{\theta}(x, y) \\
& f_{m}(x, y)=k x \cos \theta_{m}+k y \sin \theta_{m}+\varepsilon_{m} \\
& \psi_{\theta}(t)=\sigma t+\varphi \\
& \varphi=\tan ^{-1} \frac{\sum_{m=1}^{M} a_{m} \sin \left\{f_{m}(x, y)\right\}}{\sum_{m=1}^{M} a_{m} \cos \left\{f_{m}(x, y)\right\}}
\end{aligned}
$$

where

Then the wave profile equation of Eq. 10 in the case of the multi-directional wave is rewritten as follows.

$$
\eta_{\theta}(x, y, t)=r_{\theta}(x, y) \cos (\sigma t+\varphi)
$$

The envelope surface $r_{\theta}(x, y)$ is not varied in time, i.e., it is time-invariant. Therefore, the directional wave has a constant envelope surface in terms of time $t$.

Here, to show simple directional wave, let us consider the "phase" wave in which the amplitude of each com. ponent wave is the same value as follows;

$$
\begin{aligned}
\eta_{\theta}(x, y, t)= & \sum_{m=0}^{M} a \cos \{\sigma t+k x \cos (\theta+m \cdot \Delta \theta) \\
& +k y \sin (\theta+m \cdot \Delta \theta)\}
\end{aligned}
$$

Equation 20 can be modified

$$
\eta_{\theta}(x, y, t)=\frac{\sin \frac{(M+1) \beta}{2}}{\sin \frac{\beta}{2}} \cdot a \cos \left(\alpha+\frac{M}{2} \beta\right)
$$

where

$$
\begin{aligned}
& \alpha=\sigma t+k x \cos \theta+k y \sin \theta \\
& \beta=k \Delta \theta(-x \sin \theta+y \cos \theta)
\end{aligned}
$$

\section{2 "Directional" swell}

In the case of the multi-directional wave, a "directional" grouping wave must be discussed. A grouping wave of section 2 could be called a "frequency" grouping wave in which multiple frequency component waves exist. However, a "directional" grouping wave has just a single frequency, but multiple directional component waves exist in it.

So-called a "directional" swell is a simple case of a "directional" grouping wave. In Eq. 10 , let $M=2, a_{1}=a_{2}$ $=a$ and $\varepsilon_{1}=\varepsilon_{2}=0$, in a similar way of a "frequency" swell, then it follows that

$$
\begin{aligned}
\eta_{\theta}(x, y, t) & =a \cos \left(\sigma t+k x \cos \theta_{1}+k y \sin \theta_{1}\right) \\
& +a \cos \left(\sigma t+k x \cos \theta_{2}+k y \sin \theta_{2}\right) \\
& =2 a \cos \left\{\frac{1}{2} k x\left(\cos \theta_{1}-\cos \theta_{2}\right)\right.
\end{aligned}
$$




$$
\begin{aligned}
& \left.+\frac{1}{2} k y\left(\sin \theta_{1}-\sin \theta_{2}\right)\right\} \\
& \quad \times \cos \left\{\sigma t+\frac{1}{2} k x\left(\cos \theta_{1}+\cos \theta_{2}\right)\right. \\
& \left.+\frac{1}{2} k y\left(\sin \theta_{1}+\sin \theta_{2}\right)\right\}
\end{aligned}
$$

The first cosine function of the second expression of Eq. 24 corresponds to the wave envelope surface which is not varied in time and the second cosine function presents the carrier surface of the wave $\eta_{\theta}(x, y, t)$ which is time variant.

Figure 4 demonstrates the surface of a "directional" swell of which the angular frequency is $0.57[\mathrm{rad} / \mathrm{sec}]$ and the component directions are $\pi / 2$ [rad] and $\pi / 3$ [rad]. Figure 5 shows the decomposed surfaces: the wave envelope surface and the carrier surface of this "directional" swell.

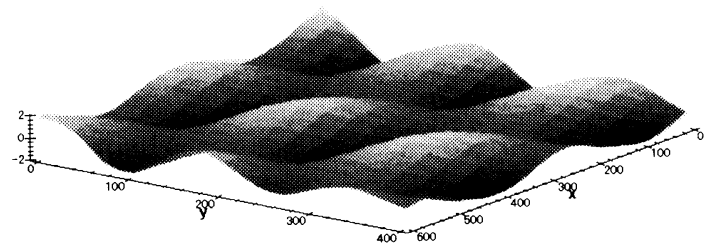

Fig. 4 Surface of a "directional" swell of which the angular frequency is $0.57[\mathrm{rad} / \mathrm{sec}]$ and the component directions are $\pi / 2[\mathrm{rad}]$ and $\pi / 3[\mathrm{rad}]$.

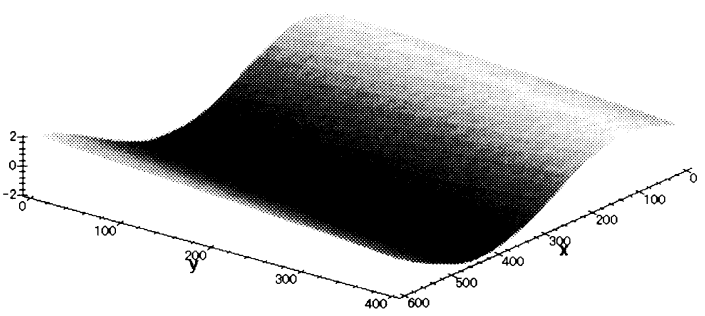

WAVE ENVELOPE SURFACE

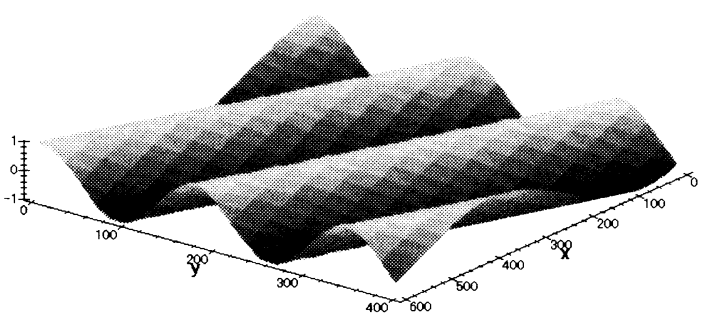

CARRIER SURFACE

Fig. 5 Decomposed surfaces: the wave envelope surface and the carrier surface of Fig. 4 (A "directional" swell).

\section{4. “Complex" Grouping Wave}

\section{1 Multi-directional irregular wave and its enve- lope}

The surface profile $\eta_{\sigma \theta}(x, y, t)$ of the multi-directional irregular ocean wave, so-called the "short crested" wave, is generally written by

$$
\begin{array}{r}
\eta_{\sigma \theta}(x, y, t)=\sum_{n=1}^{N} \sum_{m=1}^{M} a_{n m} \cos \left(\sigma_{n} t+k_{n} x \cos \theta_{m}+\right. \\
\left.k_{n} y \sin \theta_{m}+\varepsilon_{n m}\right)
\end{array}
$$

Here,

$$
a_{n m}=\sqrt{2 S\left(\sigma_{n}, \theta_{m}\right) \Delta \sigma_{n} \Delta \theta_{m}}
$$

$S(\sigma, \theta)$ means the directional spectrum. This is the mostly common wave which is appeared in the real ocean.

The result of the formulation of the wave envelope and the carrier in this case is only shown as follows: Product form of the wave envelope and the carrier

$$
\eta_{\sigma \theta}(x, y, t)=r_{\sigma \theta}(x, y, t) \cos \psi_{\sigma \theta}(x, y, t)
$$

Wave envelope surface (amplitude modulation)

$$
\begin{aligned}
& \eta_{\sigma \theta}(x, y, t)=\sqrt{\eta_{\sigma \theta}^{2}(x, y, t)+\xi_{\sigma \theta}^{2}(x, y, t)} \\
& \xi_{\sigma \theta}(x, y, t)=\frac{1}{\pi} \int_{-\infty}^{\infty} \frac{\eta_{\sigma \theta}(x, y, \tau)}{t-\tau} d \tau
\end{aligned}
$$

Phase modulation

$$
\psi_{\sigma \theta \theta}(x, y, t)=\tan ^{-1} \frac{\xi_{\sigma \theta}(x, y, t)}{\eta_{\sigma \theta}(x, y, t)}
$$

\section{2 "Complex" swell}

A "complex" grouping wave has a special directional spectrum which has low frequency and concentrated propagation direction compared to the primary directional spectrum.

A "complex" swell is a simple case of a "complex" grouping wave. In a similar way of a "frequency" swell, the equation is written by

$$
\begin{aligned}
\eta_{\sigma \theta}(x, y, t)= & a \cos \left(\sigma_{1} t+k_{1} x \cos \theta_{1}+k_{1} y \sin \theta_{1}\right) \\
& +a \cos \left(\sigma_{2} t+k_{2} x \cos \theta_{2}+k_{2} y \sin \theta_{2}\right) \\
= & 2 a \cos \left\{\frac{\sigma_{1}-\sigma_{2}}{2} t+\frac{x}{2}\left(k_{1} \cos \theta_{1}\right.\right. \\
& \left.-k_{2} \cos \theta_{2}\right)+\frac{y}{2}\left(k_{1} \sin \theta_{1}\right. \\
& \left.\left.-k_{2} \sin \theta_{2}\right)\right\} \times \cos \left\{\frac{\sigma_{1}+\sigma_{2}}{2} t\right. \\
& +\frac{x}{2}\left(k_{1} \cos \theta_{1}+k_{2} \cos \theta_{2}\right) \\
& \left.+\frac{y}{2}\left(k_{1} \sin \theta_{1}+k_{2} \sin \theta_{2}\right)\right\}
\end{aligned}
$$

In this case, both the wave envelope surface and the carrier surface are time variant and also directional dependent. Figure 6 shows the surface of a "complex" swell of which the angular frequencies and the component directions are $0.314[\mathrm{rad} / \mathrm{sec}], 0.57[\mathrm{rad} / \mathrm{sec}]$ and $\pi / 2[\mathrm{rad}], \pi / 3$ [rad], respectively. Figure 7 represents the decomposed surfaces: the wave envelope surface and the carrier surface of this "complex" swell.

\section{3 Consideration of phase plane of the envelope}

Let us consider the characteristics of the phase plane of the envelope. It is shown that the phase plane of the envelope of a "complex" wave is superposed by the 


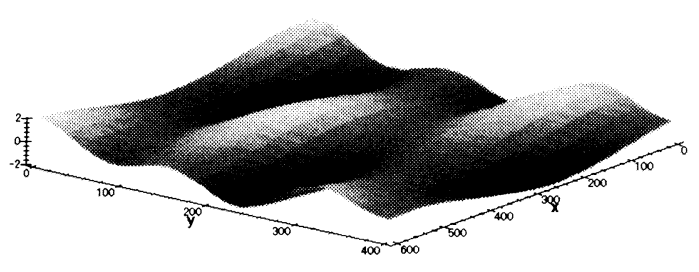

Fig. 6 Surface of a "complex" swell of which the angular frequencies and the component directions are $0.314[\mathrm{rad} / \mathrm{sec}], 0.57[\mathrm{rad} / \mathrm{sec}]$ and $\pi / 2$ [rad], $\pi / 3[\mathrm{rad}]$, respectively.

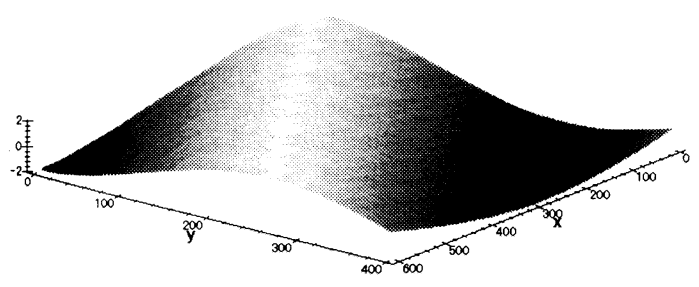

WAVE ENVELOPE SURFACE

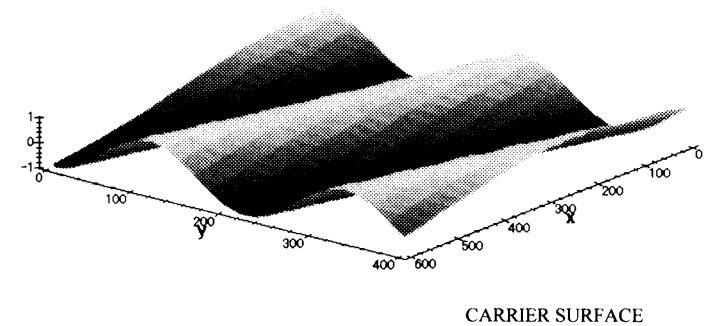

Fig. 7 Decomposed surfaces: the wave envelope surface and the carrier surface of Fig. 6 (A "complex" swell).

phase planes of the envelopes of a "frequency" swell and a "directional" swell.

The phase planes of the envelopes of a "frequency" swell and a "directional" swell are considered from Eq. 9 and Eq. 24. Let the original suffix 1 and 2 be a meaning of primary and secondary (denoted by') values of each variable, respectively, it follows that

$$
\begin{aligned}
& \frac{k-k^{\prime}}{2} x \cos \theta+\frac{k-k^{\prime}}{2} y \sin \theta \\
& \text { for a "frequency" swell } \\
& \frac{1}{2} k x\left(\cos \theta-\cos \theta^{\prime}\right)+\frac{1}{2} k y\left(\sin \theta-\sin \theta^{\prime}\right) \\
& \text { for a "directional" swell }
\end{aligned}
$$

The summation of Eq. 32 and Eq. 33 is as follows :

$$
\begin{aligned}
& \frac{k-k^{\prime}}{2} x \cos \theta+\frac{k-k^{\prime}}{2} y \sin \theta+\frac{1}{2} k x\left(\cos \theta-\cos \theta^{\prime}\right) \\
& +\frac{1}{2} k y\left(\sin \theta-\sin \theta^{\prime}\right)
\end{aligned}
$$

On the other hand, from Eq. 31, the phase plane of the envelope of a "complex" swell is $\frac{x}{2}\left(k \cos \theta-k^{\prime} \cos \theta^{\prime}\right)+\frac{y}{2}\left(k \sin \theta-k^{\prime} \sin \theta^{\prime}\right)$ for a

"complex" swell

Based on the assumption of $k^{\prime}=k+\Delta k$ ( $\Delta k$ is very small compared to $k$ ) and $\theta^{\prime}=\theta+\Delta \theta$ ( $\Delta \theta$ is very small compared to $\theta$, similarly), Eq. 34 and Eq. 35 nearly equal as follows:

$$
\frac{x}{2}(k \Delta \theta \sin \theta-\Delta k \cos \theta)-\frac{y}{2}(k \Delta \theta \cos \theta+\Delta k \sin \theta)
$$

That is, the phase plane of the envelope of a "complex" wave is superposed by the phase planes of the envelopes of a "frequency" swell and a "directional" swell.

\section{Concluding Remark}

In this paper, some types of the grouping wave are reduced and their characteristics are discussed. The normal grouping wave ("frequency" grouping wave) has the time varying envelope surface. However, the wave envelope surface of the "directional" grouping wave is not varied in time, i. e., it is time-invariant. Therefore, the "directional" grouping wave has a constant envelope surface of time. Moreover, the complex grouping wave is reduced from the multi-directional irregular wave. It is shown that the phase plane of the envelope of a "complex" wave is superposed by the phase planes of the envelopes of a "frequency" swell and a "directional" swell.

From the consideration of this paper the followings are concluded.

(1) The "directional" grouping wave does not cause low-frequency motions to floating structures because the wave envelope surface of it is timeinvariant. However, the structure design for it will have to be re-considered due to the timeinvariant, long duration envelope.

( 2 ) The "complex" grouping wave also causes lowfrequency motions and the structure design for it will have to be re-considered too because the "complex" grouping wave has the characteristics of both the "frequency" grouping wave and the "directional" grouping wave.

The grouping wave can cause severe motion in floating structures such as semi-submersible drilling rigs, floating airports or vessels as well, owing to its frequency being near resonance, and it can interfere with other operations both offshore and on the coast. There is therefore considerable interests in physical and engineering studies. The author considers the continuing research of the grouping wave is required to analyze the characteristics of it and to have a suitable design method for mooring systems.

Note: Two approximated equations are used.

$k^{\prime} \cos \theta^{\prime} \approx k \cos \theta+\Delta k \cos \theta-k \Delta \theta \sin \theta$

$k^{\prime} \sin \theta^{\prime} \approx k \sin \theta+\Delta k \sin \theta+k \Delta \theta \cos \theta$ 


\section{Acknowledgment}

The author would like to thank a reviewer for helpful and critical suggestions.

\section{References}

1) Goda, Y., "On the Statistics of Wave Groups," Report of the Port and Harbour Research Institute, 15, 3, (1976) pp. 3-19. (in Japanese)

2) Longuet-Higgins, M. S. "On the distribution of the heights of sea waves: some effects of nonlinearity and finite band -width," J. Geophysical Res. 85, (C3), (1980) pp. 1519-1523.

3) Ottsen-Hansen, N. E., Sand, S. E., Lundgren, H., Sorensen, T., and Gravesen, H., "Correct Reproduction of Group-induced long wave," Proc. Int'l Conf. Coastal Engineering, (1980) pp. 784-800.

4) Mansard, E. P. D. and Barthel, V., "Shoaling Properties of Bounded Long Wave,” ibid., (1984) pp. 798-814.

5) Velcheva, A.D. and Cherneva, Z. I., "The Influence of Wave Grouping on Wave Height Distribution," Proc. of the 17th Int'l Conf. on Offshore Mechanics and Arctic Engineering, OMAE98-1480, (1998)

6) Hiraishi, T., Tadokoro, A., and Fujisaku, H.,
"Characteristics of Long Period Wave Observed in Port," Report of the Port and Harbour Research Institute, 35, 3, (1996) pp. 3-35. (in Japanese)

7) Research Report of the Mega-float, Mega-float Research Association, (1996). (in Japanese)

8) Naito, S., "Generation of an Irregular Wave and Its Randomness," Seminar on Stability of Ship \& Offshore Structures, Pusan, Korea, (1993).

9) Nohara, B. T. and Matsuura, M., "Generation of the Irregular Wave Specified by the Spectrum of the Wave Envelope and Some Results of a Model Test," Journal of Soc. of Naval Arch. of Japan, 183, (1998) pp. 115-121.

10) Nohara, B. T., "Consideration of Non-cyclic Trajectory of Grouping Wave," Proc. of the 42nd Annual Conf. of the Institute of Systems, Control and Information Engineers, (1998) pp. 117-118.

11) For example, Crapper, G. D., "Introduction to Water Waves,” Ellis Horwood Ltd., (1984).

12) Hahan, S. L., "Hilbert Transforms in Signal Processing," Artech House, (1996).

13) Barber, N.F. and Ursell, F., "The generation and propagation of ocean waves and swell: 1 wave periods and velocities," Phil. Trans. Roy. Soc. A, 240. (1984) pp. 527-560. 